\title{
A microbiological multi-plate method to detect cephalosporin residues in milk
}

\author{
Pavlína Navrátilová, Jana Vyhnálková, Zachovalová Hana, Lenka Vorlová \\ University of Veterinary and Pharmaceutical Sciences Brno, Faculty of Veterinary Hygiene and Ecology, \\ Department of Milk Hygiene and Technology, Brno, Czech Republic \\ Received January 13, 2020 \\ Accepted April 30, 2020
}

\begin{abstract}
The aim of this study was to determine the detection capability $(C C \beta)$ of a multi-plate diffusion method for selected cephalosporins for which the maximum residue limits (MRLs) in milk have been set (ceftiofur, cefoperazone, cephalexine, cephazoline, cephalonium, cephapirine, cefquinome). The multiplate method was composed of Bacillus subtilis BGA CCM 4062 plates (agar $\mathrm{pH}$ of 6, 8 and 7.2); Kocuria rhizophila CCM 552 plate, Geobacillus stearothermophilus CCM 5965 plate and Escherichia coli CCM 7372 plate. Geobacillus stearothermophilus plate showed the highest sensitivity. $C C \beta$ for the tested cephalosporins was determined at MRLs or lower, except for cefquinome, which was undetectable even at $12 \times$ the MRL. Kocuria rhizophila plate showed the highest sensitivity to ceftiofur $(C C \beta=100 \mu \mathrm{g} / \mathrm{l})$; cephazoline and cephalonium could not be detected even at $12 \times$ times the MRL. Escherichia coli plate was most sensitive to cefoperazone $(C C \beta=60 \mu \mathrm{g} / \mathrm{l})$. Other than cephapirine, none of the cephalosporins could be detected by $B$. subtilis BGA $(\mathrm{pH} 6,8$ and 7.2) plates even at $12 \times$ the MRL. Our results demonstrate the differences in sensitivity to selected cephalosporins of the individual plate methods that together form the multi-plate method. The multi-plate method is sensitive enough to detect most of the tested cephalosporins, with the exception of cefquinome, which could not be detected at levels close to MRL.
\end{abstract}

Antimicrobials, detection, microbiological method

Antimicrobials are essential for the treatment and prevention of infectious diseases in food-producing animals and in protecting public health from food-borne diseases. Antimicrobials are widely used for therapeutic, metaphylactic, and prophylactic purposes. According to the OIE annual report for 2018, antimicrobials are still used for growth promotion in some countries (OIE 2018a). A complete ban on the use of antibiotics as growth promoters was instituted in the EU in 2006. Excessive and improper use of antibiotics in food animals can lead to the selection and spread of antibiotic-resistant micro-organisms and consequently, their transmission to humans via food or other routes (Schwarz et al. 2001; WHO AGISAR 2017; Aidara-Kane et al. 2018; OIE 2018a). Antimicrobial resistance is a major public health and food-safety problem (WHO AGISAR 2017). Moreover, the inappropriate and excessive use of antibiotics can lead to residues of these compounds and their metabolites being retained in edible tissues. Such residues constitute toxicological, microbiological, immunological or pharmacological public health hazards (Botsoglou and Fletouris 2001). Residues of antimicrobials in raw materials of animal origin also pose a risk to the processing industry. The presence of antibiotic residues in milk can have bactericidal or bacteriostatic effects on starter cultures used in the production of fermented dairy products and cheeses (Heeschen and Blüthgen 1991). One of the main priorities for the European Commission is to achieve the highest possible level of consumer health protection (EC - White Paper on Food Safety 2000). Over the years, a number of legislative measures have been adopted to regulate the presence of veterinary medicinal products in raw materials of animal origin (Council Directive 96/23/EC 1996, EP and Council Regulation 470/2009, Commission Regulation 37/2010, etc.). According 
to the European Medicines Agency (EMA 2008), cephalosporins are one of the groups of antimicrobials of particular public health concern. Cephalosporins are antimicrobials belonging to the beta-lactam group (EMA 2012). Cephalosporins are widely used in human medicine. In hospitals, $3^{\text {rd }}$ and $4^{\text {th }}$ generation cephalosporins are used to treat, among others, septicaemia, meningitis, hospital acquired pneumonia, intra-abdominal infections and complicated urinary tract infections. Cephalosporins have also been authorized for use in food producing animals (EMA 2008). In veterinary practice, cephalosporins are used in the treatment of septicaemias, respiratory infections, and mastitis (OIE 2018b). The use of cephalosporins was found to be associated with a risk of selection of bacteria carrying the ESBL and $A m p C$ genes in treated animals with potential implications for public health (EMEA 2008). Therefore, $3^{\text {rd }}$ and $4^{\text {th }}$ generation cephalosporins were classified as highest priority critically important antimicrobials (WHO categorization) or as veterinary critically important antimicrobials (VCIA; OIE categorization). These restricted antimicrobials should be used for the treatment of clinical conditions only when there are no alternative lower category antimicrobials that are effective. Administration should be based on the results of antimicrobial susceptibility tests. First and $2^{\text {nd }}$ generation cephalosporins are classified as highly important antimicrobials or VCIA (EMA 2019).

The presence of antimicrobial residues in raw materials and foodstuff of animal origin must be regulated to protect consumer health. As the number of antimicrobials both authorized to treat food animals and for which the maximum residue limits (MRLs) in raw materials of animal origin have been established has increased, methods of detecting residues of these substances have been developed over the last decades (Commission Decision 2002/657/EC). Screening methods are critical for monitoring antimicrobial residues in raw materials of animal origin. Screening methods are defined as "methods used to detect the presence of an analyte or class of analytes at the level of interest" in Commission Decision 2002/657/EC. Microbiological screening methods have been used to detect residues of antimicrobials for several decades (Mitchell et al. 1998) due to several advantages such as low cost, ability to detect a wide range of substances and ease of application. Among them, multi-plate diffusion methods are important as both screening and postscreening methods. The first multi-plate method was designed by Bogaerts and Wolf (1980). Nevertheless, a series of other plate diffusion methods have been developed and validated since then (Pikkemaat 2009) to ensure the highest sensitivity to a wide range of substances for monitoring established MRLs. Although plate diffusion methods are used in many countries, they have not been standardized and therefore their sensitivity may vary. Most plate diffusion methods have been developed primarily for the determination of antimicrobial residues in tissues and organs, less so in milk. The detection ability of the plate methods can be affected by a number of factors: test agar, test strains used, agar $\mathrm{pH}$, agar thickness, incubation temperature, sample application method and sample amount (Botsoglou and Fletouris 2001).

According to a survey conducted by the Federation of Veterinarians of Europe (FVE), the most common indication for the use of antimicrobials in dairy cows is mastitis and treatment for mastitis during the dry period. Because $3^{\text {rd }}$ and $4^{\text {th }}$ generation cephalosporins do not have a withdrawal time for milk, they are overused in lactating dairy cattle, often in the absence of a clear diagnosis (FVE 2016). The EU legislation (Commission Regulation 37/2010) sets maximum residue limits (MRLs) in milk for a number of cephalosporins. In the Czech Republic, the multi-plate method is used to detect antimicrobials as part of quality-control testing of milk, during routine veterinary hygiene inspections and monitoring of contaminants. It is critical that screening methods are capable of detecting antimicrobials at concentrations $\leq$ MRL.

Our study examined cephalosporins which are increasingly becoming the object of interest in veterinary medicine. The aim of our study was to determine the detection 
capability $(C C \beta)$ of the multi-plate diffusion method for selected cephalosporins from different generations (ceftiofur, cefoperazone, cephalexine, cephazoline, cephalonium, cephapirine, cefquinome) for which MRLs in milk are established.

\section{Materials and Methods}

Antimicrobial standards

The following analytical standards were purchased to prepare standard antibiotic solutions: cefoperazone sodium (CMS8039, HiMedia Laboratories, Pvt. Ltd., Mumbai, India), cephalexin hydrate (CMS 647, HiMedia), ceftiofur sodium (PHR1521, Sigma Aldrich INC, St. Luis, USA), cephazoline (CMS 650 HiMedia), ciprofloxacin (17850, Fluka TM Germany), trimethoprim (T-7883 Sigma Aldrich), sulphadimidine (CMS 8933 HiMedia), cephapirin sodium (43989, Sigma Aldrich), cefquinome sulfate (32472 Sigma Aldrich), cephalonium hydrate (32904 Sigma Aldrich) and penicillin G sodium salt (TC020 HiMedia).

\section{Test strains}

Test strains used in the plate diffusion method were Bacillus subtilis BGA CCM 4062, Kocuria rhizophila CCM 552 (ATCC 9341), Geobacillus stearothermophilus CCM 5965 (ATCC 10149) and Escherichia coli CCM 7372 (ATCC 11303). The test strains for plate diffusion methods were obtained from the Czech Collection of Microorganisms (Faculty of Natural Sciences, Masaryk University, Brno, Czech Republic).

Agar plate and cultivation broth composition

The following basic media were used to prepare test plates: antimicrobial inhibitor test agar pH 6.0 (M1631 HiMedia Laboratories Pvt. Ltd. India), antimicrobial inhibitor test agar pH 8.0 (M1632 HiMedia), antimicrobial inhibitor test agar pH 7.2 (M 1601, HiMedia). The test agar medium for plates used to grow G. stearothermophilus contained: $2.5 \mathrm{~g}$ yeast extract (LP 0021, Thermo Fisher Specialty Diagnostics Ltd, UK), 5.0 g casein enzyme hydrolysate (RM 014 HiMedia), $1.0 \mathrm{~g}$ D-glucose monohydrate (49159, Fluka ${ }^{\mathrm{TM}}$ ), $15.0 \mathrm{~g}$ bacteriological agar no. 1 (LP 0011, Thermo Fisher Specialty Diagnostics Ltd), and up to $1000 \mathrm{ml}$ distilled water (sterilized for $15 \mathrm{~min}$ at $121{ }^{\circ} \mathrm{C}, \mathrm{pH} 8 \pm 0.1$ ). To cultivate $K$. rhizophila we used the brain heart infusion medium (CM1135, Thermo Fisher Specialty Diagnostics Ltd.) and the cultivation medium for G. stearothermophilus contained: $10.0 \mathrm{~g}$ yeast extract (LP 0021, Thermo Fisher Specialty Diagnostics Ltd), $20.0 \mathrm{~g}$ casein enzyme hydrolysate (RM 014 HiMedia), $0.5 \mathrm{~g} \mathrm{D}$-glucose monohydrate, and up to $1000 \mathrm{ml}$ distilled water (sterilized for $15 \mathrm{~min}$ at $121{ }^{\circ} \mathrm{C}, \mathrm{pH} 8 \pm 0.1$ ).

Six-plate diffusion method

The 6-plate diffusion method was carried out in accordance with the guidelines issued by the Czech State Veterinary Administration - National Reference Laboratory for Mycotoxins and Other Natural Toxins, Dyes and Antibacterial (Inhibiting) Substances and Residues of Veterinary Drugs (SVA 2008).

Method using Bacillus subtilis BGA CCM 4062 (agar pH 6.0;8.0 and 7.2)

The test agar ( $\mathrm{pH} 6.0, \mathrm{pH} 8.0$ a $\mathrm{pH} 7.2$ ) was cooled to $45-50{ }^{\circ} \mathrm{C}$ and was inoculated with a suspension of B. subtilis BGA CCM 4062. A solution of trimethoprim $(c=5 \mu \mathrm{g} / \mathrm{ml})$ was added to the $\mathrm{pH} 7.2$ agar medium. Four $\mathrm{ml}$ of the test agar medium were poured into each $90 \mathrm{~mm}$ diameter Petri dish and incubated at $30^{\circ} \mathrm{C}$ for 18-24 h.

\section{Method using Kocuria rhizophila CCM 552}

The organism was cultured according to the supplier's instructions. The cultivation broth was inoculated with a 24-h-old suspension and incubated at $37^{\circ} \mathrm{C}$ for $2 \mathrm{~h}$. This culture was then used to inoculate the test agar medium at $\mathrm{pH} 8.0$ cooled to $45-50{ }^{\circ} \mathrm{C}$. Four $\mathrm{ml}$ of the test agar medium were poured into each $90 \mathrm{~mm}$ diameter Petri dish and incubated at $37^{\circ} \mathrm{C}$ for $18-24 \mathrm{~h}$.

\section{Method using Geobacillus stearothermophilus CCM 5965}

The organism was cultured according to the supplier's instructions. The cultivation broth was inoculated with the test strain and incubated at $55^{\circ} \mathrm{C}$ for $2 \mathrm{~h}$. This culture was then used to inoculate the agar medium at $\mathrm{pH} 8$. Four $\mathrm{ml}$ of the test agar medium were poured into each $90 \mathrm{~mm}$ diameter Petri dish and incubated at $55^{\circ} \mathrm{C}$ for 18-24 h. The method using E. coli CCM 7372 was published previously (Navrátilová et al. 2014).

Testing the sensitivity of the multi-plate method

The sensitivity check of the prepared plate methods was checked in accordance with the methodological guideline (SVA 2008) by applying $10 \mu \mathrm{l}$ of a working solution of the specific antibiotic to a sterile 6 mm diameter disc. The type and concentration of the antibiotics, as well as the size of the inhibition zones are shown in Table 1.

\section{Determination of detection limits}

Working solutions were prepared from standard antibiotic solutions with a final substance concentration $c=1 \mathrm{mg} \cdot \mathrm{ml}^{-1}$ by dilution with distilled water. Spiked milk samples were prepared by diluting the calculated 
Table 1. Testing the sensitivity of the plate method.

\begin{tabular}{llc}
\hline Concentration of antibiotics & \multicolumn{1}{c}{ Plate method } & Size of inhibition zone (mm) \\
\hline Penicillin $1 \mathrm{IU} / \mathrm{ml}$ & Bacillus subtilis BGA pH 6.0 & $6.0 \pm 1.5$ \\
Streptomycin $100 \mu \mathrm{g} / \mathrm{ml}$ & Bacillus subtilis BGA pH 8.0 & $8.0 \pm 1.5$ \\
Streptomycin $100 \mu \mathrm{g} / \mathrm{ml}$ & Kocuria rhizophila CCM 552 & $6.0 \pm 1.5$ \\
& Bacillus subtilis BGA pH 7.2 & $6.0 \pm 1.5$ \\
Penicillin $1 \mathrm{IU} / \mathrm{ml}$ & Geobacillus stearothermophilus CCM 5965 & $11.0 \pm 1.5$ \\
Ciprofloxacin $0.3(\mu \mathrm{g} / \mathrm{ml})$ & Escherichia coli CCM 7372 & $5.5 \pm 1.5$ \\
\hline
\end{tabular}

amount of cephalosporin working solution with milk (pasteurized milk, $1.5 \%$ fat), which was simultaneously tested for the presence of antimicrobials as a negative control. The artificially prepared spiked milk samples with a defined cephalosporin concentration were agitated and applied (100 $\mu \mathrm{l})$ to a sterile $12.7 \mathrm{~mm}$ diameter disc (Blanc paper discs, Albet ${ }^{\mathbb{B}}$ LabScience, Barcelona, Spain). The final concentrations of cephalosporins in the spiked milk samples for each method are given in Table 2. Each spiked sample with baseline antibiotic concentrations of $0.5 \times, 1 \times$, and $1.5 \times$ MRLs were tested $20 \times$, and the final determination of $C C \beta$ detection level was carried out $60 \times$. Following incubation of the plates under defined conditions, the final inhibition of bacterial growth around the disc (inhibition zone; IZ) was evaluated, and its size in mm was recorded. The width of the IZ is defined as the distance of the outer edge of the inhibition zone from the edge of the disc. IZ size was measured using a special ruler (HiAntibiotic Zone Scale, HiMedia). A regular IZ of $\geq 2 \mathrm{~mm}$ was interpreted as a positive (suspect) test result for $B$. subtilis, $K$. rhizophila and $E$. coli. In the method with $G$. stearothermophilus, an IZ size $\geq 1 \mathrm{~mm}$ was interpreted as a suspect result. The sensitivity of the plates was determined in accordance with the requirements of EC legislation (Commission Decision 2002/657/EC), based on $C C \beta$. Detection capability is defined in the Annex to Commission Decision 2002/657/EC as "the smallest content of the analyte that may be detected, identified and/or quantified in a sample with an error probability of $\beta$. In the case of analytes with an established MRL, $C C \beta$ is the concentration at which the method is able to detect permitted limit concentrations with a statistical certainty of $1-\beta$. $C C \beta$ is the concentration at which only $\leq 5 \%$ false compliant results remain. In this case, $C C \beta$ must be less than or equal to the MRL".

Table 2. Range of spiked milk concentrations $(\mu \mathrm{g} / 1)$.

\begin{tabular}{lcccc}
\hline & \multicolumn{3}{c}{ Plate method } \\
\cline { 2 - 5 } Cephalosporin & $\begin{array}{c}\text { Bacillus subtilis BGA } \\
\mathrm{pH} 6.0,7.2,8.0\end{array}$ & $\begin{array}{c}\text { Kocuria rhizophila } \\
\text { CCM 552 }\end{array}$ & $\begin{array}{c}\text { Geobacillus stearothermophilus } \\
\text { CCM 5965 }\end{array}$ & $\begin{array}{c}\text { Escherichia coli } \\
\text { CCM 7372 }\end{array}$ \\
\hline Ceftiofur & $50-1200$ & $50-150$ & $50-150$ & $50-530$ \\
Cefoperazone & $25-600$ & $25-500$ & $25-75$ & $25-75$ \\
Cephalexine & $50-1200$ & $50-500$ & $50-150$ & $50-1200$ \\
Cephazoline & $25-600$ & $25-600$ & $25-75$ & $25-600$ \\
Cephalonium & $10-240$ & $10-240$ & $10-30$ & $10-240$ \\
Cephapirine & $30-120$ & $30-280$ & $30-90$ & $30-720$ \\
Cefquinome & $10-240$ & $10-170$ & $10-240$ & $10-140$ \\
\hline
\end{tabular}

\section{Results}

From the results in Table 3 it is evident that the plate method with G. stearothermophilus CCM 5965 showed the highest sensitivity to the tested cephalosporins. The method reliably detected all the tested cephalosporins (except cefquinome) at a MRL level or lower. The method was most sensitive for cephazoline, cephapirine (both $C C \beta \geq 0.2 \times \mathrm{MRL}$ ) and cefoperazone $(C C \beta \geq 0.8 \times \mathrm{MRL})$. The method showed a $C C \beta$ at $1 \times \mathrm{MRL}$ level for determining residues of ceftiofur, cephalexine and cephalonium. Cefquinome residues could not be detected. The method with the $K$. rhizophila CCM 552 showed the highest sensitivity to ceftiofur $(C C \beta \geq 1 \times \mathrm{MRL})$. The method was able to detect residues of cefoperazone, cephalexine, cephapirine and cefquinome at concentrations many times higher than the established MRL $(C C \beta \geq 4.6-10 \times \mathrm{MRL})$. Two cephalosporins (cephazoline and cephalonium) could not be detected by this method even at concentrations equal to $12 \times$ MRL. 


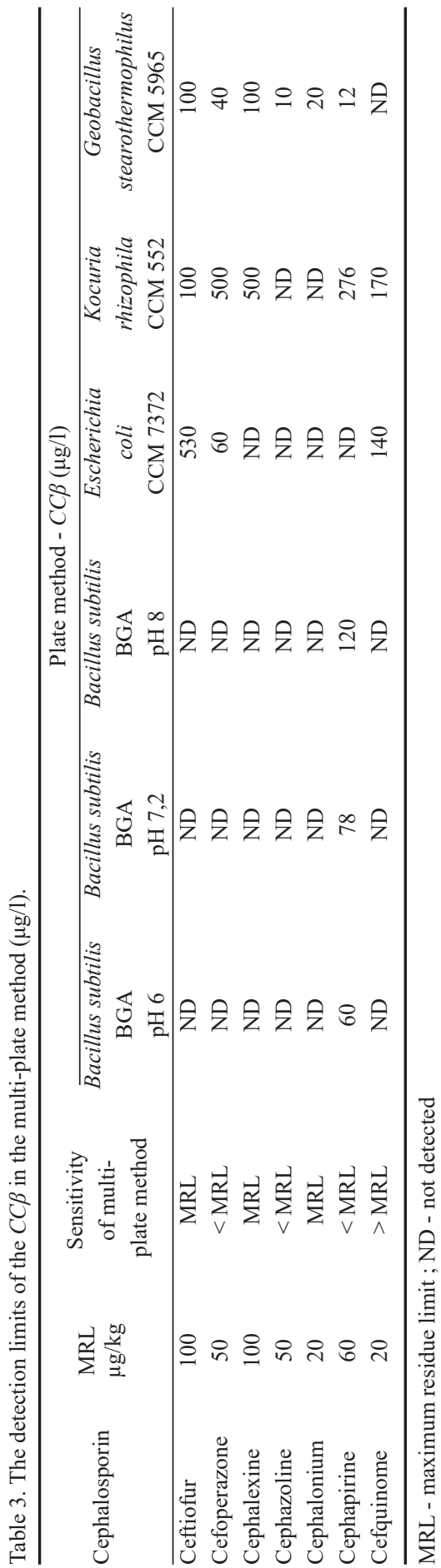

The method with E. coli CCM 7372 was sensitive only to 3 cephalosporins: the detection capability for ceftiofur and cefquinome was much higher than the established MRL $(C C \beta \geq 5.3 \times \mathrm{MRL}$ and $C C \beta \geq 7 \times \mathrm{MRL}$, respectively) and for cefoperazone it was slightly higher than the set MRL $(C C \beta \geq 1.2$ $\times$ MRL). Four cephalosporins (cephazoline, cephalexine, cephalonium, cephapirine) could not be detected even at high concentrations, the method being unable to detect concentrations equal to $12 \times$ MRL. Table 3 also shows that among the tested cephalosporins, only cephapirine was detected with $B$. subtilis (pH 6.0, 8.0, and 7.2). The detection capability of these 3 methods with $B$. subtilis for cephapirine was in the concentration range of 1-2 $\times$ MRL. The rest of the tested cephalosporins could not be detected even at concentrations equal to $12 \times$ MRL.

\section{Discussion}

In the Czech Republic, the multi-plate diffusion method is the reference method for the determination of antimicrobial residues in raw materials and foodstuffs of animal origin (SVA 2008). The multi-plate method consists of 6 plate methods. Some European countries employ the STAR method, designed and validated by Gaudin et al. (2004). This multi-plate method comprises 5 plates with test strains of $B$. subtilis BGA pH 7.2, Kocuria varians ATCC 9341 pH 8, Bacillus cereus ATCC 11778 pH 6, E. coli ATCC 11303 and Bacillus stearothermophilus ATCC 10149. Among cephalosporin antibiotics the authors assessed sensitivity of the method to cephazoline, cephapirine, cephalonium, ceftiofur, cephacetrile, cefquinome, cephalexin and cefoperazone. When validating the method, they found that the STAR method was able to detect 2 cephalosporins at $\leq$ MRL concentrations. It showed very good sensitivity to ceftiofur $(125 \mu \mathrm{g} / \mathrm{l})$ and cephalexine (25-30 $\mu \mathrm{g} / \mathrm{l})$. For the other four cephalosporins (cephapirine, cephalonium, cefquinome, cefoperazone), the sensitivity of the method was in the range of $1-4 \times$ MRL. The remaining 2 cephalosporins, cephazoline and cephacetrile, were detected only at concentrations greater than $4 \times$ MRL. In the multi-plate method 
presented here, we were able to detect cefoperazone, cephazoline and cephapirine at concentrations $<$ MRL, and ceftiofur, cephalexine and cephalonium at the MRL level. The method was not sufficiently sensitive only for cefquinome. The sensitivity of the methods is affected by the varied protocols followed (different number of plates, different test strains, different agar media, different amount of applied sample, etc.). Furthermore, for some cephalosporins, the MRLs were different at the time of publication. The objective of the study published by Kirbiš (2006) was to develop a microbiological method for the detection of macrolide, aminoglycoside, cephalosporin and tetracycline antibiotics. The author used a multi-plate method consisting of a total of 5 plates with test strains of B. cereus ATTC 11778, Micrococcus luteus ATCC 9341, E. coli ATCC 10536, Staphylococcus epidermidis ATCC 12228, and B. subtilis BGA. The study tested susceptibility to 3 cephalosporins: cephazoline, cephalexine, and cefoperazone. Of the plate methods, $M$. luteus proved to be the most sensitive to cephalosporin antibiotics. The detection capability was $25 \mu \mathrm{g} / \mathrm{kg}$ for cephazoline and $50 \mu \mathrm{g} / \mathrm{kg}$ for cephalexine and cefoperazone, i.e. $\leq$ MRL according to Kirbiš (2006). Sensitivity to cephalosporins was also demonstrated with $B$. subtilis BGA. In the present study, $K$. rhizophila (formerly M. luteus) showed much lower sensitivity to cefoperazone and cephalexine $(C C \beta \geq 500 \mu \mathrm{g} / \mathrm{l})$, cephazoline could not be detected even at $12 \times$ MRL. Escherichia coli appeared to be resistant to cephalexine, cephazoline, and cefoperazone residues in the cited study (Kirbiš 2006). Also, in our study, it was not possible to detect cephalexin and cephazoline with $E$. coli, whereas ceftiofur and cefoperazone were detected at concentrations above the MRLs $(C C \beta 530 \geq \mu \mathrm{g} / 1$ and $C C \beta \geq 60 \mu \mathrm{g} / 1$, respectively). In contrast to the cited study (Kirbiš 2006), none of these cephalosporins (cephazoline, cephalexine, cefoperazone) could be detected with $B$. subtilis BGA. However, the method of plate preparation and sample testing was quite different in Kirbiš (2006). In 1999, Nouws et al. (1999) developed a multi-plate method comprising 7 plates with different test strains that could be used to detect antimicrobial residues in milk. The aim was to design a method to capture the widest possible spectrum of antibiotics at levels close to the established MRL. The plate with Bacillus stearothermophilus C 953 was the only one able to detect cephalosporin antibiotics (with the exception of cefquinome) at low concentrations (below MRLs). A positive result on this plate was recorded for $55 \mu \mathrm{g} / 1$ ceftiofur, $80 \mu \mathrm{g} / 1$ cephalexin, $55 \mu \mathrm{g} / 1$ cefoperazone and $20 \mu \mathrm{g} / 1$ cephazoline. Similarly, in our study, the plate with $G$. stearothermophilus was most sensitive to cephalosporin antibiotics. Compared to Nouws et al. (1999) the $C C \beta$ values for ceftiofur and cephalexine were higher $(100 \mu \mathrm{g} / \mathrm{l}$ and $100 \mu \mathrm{g} / \mathrm{l}$, respectively), but were lower for cefoperazone and cephazoline $(40 \mu \mathrm{g} / \mathrm{l}$ and $10 \mu \mathrm{g} / \mathrm{l}$, respectively). Moreover, the methodology for examining samples in the cited study was also different. At the time of the study (around 1999), MRLs had not been established for some cephalosporins.

Our results showed differences in sensitivity of the various test strains, comprising the multi-plate method, to selected cephalosporin group antibiotics. The most sensitive method for detecting cephalosporin residues in milk was a plate with G. stearothermophilus, which was able to detect all the tested cephalosporins (with the exception of cefquinome) at concentrations of $\leq$ MRL. In contrast, the lowest sensitivity was observed with plates using B. subtilis BGA. The detection limits of the multi-plate method are sufficient to detect most tested cephalosporins. The only one that could not be detected at levels close to MRL was cefquinome, which is registered as an intramammary veterinary pharmaceutical in the Czech Republic.

\section{Acknowledgements}

This work was supported by the Internal Grant Agency of the University of Veterinary and Pharmaceutical Sciences Brno (ITA 2340/1/FVHE 2019). 


\section{References}

Aidara-Kane A, Frederick JA, Conly JM, Minato Y, Silbergeld EK, McEwen SA, Collignon PJ and for the WHO Guideline Development Group 2018: World Health Organization (WHO) guidelines on use of medically important antimicrobials in food-producing animals. Antimicrob Resist Infect Control 7:7

Bogaerts R, Wolf F 1980: A standardized method for the detection of residues of anti-bacterial substances in fresh meat - EEC (European Communities). Fleischwirtschaft 60: 667-675

Botsoglou NA, Fletouris DI 2001: Drug Residues in Foods: Pharmacology, Food Safety, and Analysis. Marcel Dekker, New York, 1194 p.

Commission Decision 2002/657/EC of 12 August 2002, implementing Council Directive 96/23/EC concerning the performance of analytical methods and the interpretation of results. OJ L 221: 8-36

Commission Regulation (EU) No. 37/2010 of 22 December 2009: on pharmacologically active substances and their classification regarding maximum residue limits in foodstuffs of animal origin. OJ L 15: 1-72

Council Directive 96/23/EC of 29 April 1996 on measures to monitor certain substances and residues thereof in live animals and animal products and repealing Directives 85/358/EEC and 86/469/EEC and Decision 89/187/EEC and 91/664/EEC. OJ L 125: 10-32

European Medicines Agency 2008: Revised reflection paper on the use of $3^{\text {rd }}$ and $4^{\text {th }}$ generation cephalosporins in food producing animals in the European Union: development of resistance and impact on human and animal health. (EMEA/CVMP/SAGAM/81730/2006-Rev.1). Available at: www.ema.europa.eu/documents/scientificguideline/revised-reflection-paper-use-third-fourth-generation-cephalosporins-food-producing-animalseuropean_en.pdf. Last modified February 18, 2008. Accessed July 11, 2019

European Medicines Agency 2012: Veterinary medicines and product data management. Referral procedure on veterinary medicinal products containing $3^{\text {rd }}$ and $4^{\text {th }}$ generation cephalosporins under Article 35 of Directive 2001/82/EC, as amended (EMA/253066/2012). Available at: www.ema.europa.eu/en/documents/referral/ questions-answers-referral-procedure-veterinary-medicinal-products-containing-3rd-4th-generation_en.pdf. Last modified May 7, 2008. Accessed July 11, 2019

European Medicines Agency 2019: Answer to the request from the European Commission for updating the scientific advice on the impact on public health and animal health of the use of antibiotics in animals - Categorisation of antimicrobials (EMA/CVMP/CHMP/682198/2017)._Available at: www.ema.europa.eu/ en/documents/other/answer-request-european-commission-updating-scientific-advice-impact-public-healthanimal-health-use en.pdf. Last modified February 4, 2019. Accessed September 20, 2019

European Commission 2000: White Paper on Food Safety, Available at: www.ec.europa.eu/food/food/intro/ white paper en.pdf. Accessed September 9, 2019.

Federation of Veterinarians of Europe (FVE) 2016: Antimicrobial use in food-producing animals. (FVE/016/DOC/O10). Available at: www.ema.europa.eu/en/documents/report/annex-replies-efsa/ema-questions-useantimicrobialsfood-producing-animals-eu-possible-measures-reduceantimicrobial_en.pdf. Last modified February 29, 2016. Accessed September 10, 2019

Gaudin V, Maris P, Fuselier R, Ribouchon JL, Cadieu N, Rault A 2004: Validation of a microbiological method: the STAR protocol, a five-plate test, for the screening of antibiotic residues in milk. Food Addit Contam 21: 422-433

Heeschen WH, Blüthgen A 1991: Veterinary drugs and pharmacologically active compounds. In: Monograph on Residues and Contaminants in Milk and Milk Products. IDF, Brusel, pp. 13-69

Kirbiš A 2006: Microbiological 5-plate screening method for detection of tetracyclines, aminoglycosides, cephalosporins and macrolides in milk. Slov Vet Res 43: 161-168

Mitchell JM, Griffiths MW, McEwen S A, McNab WB, Yee AJ 1998: Antimicrobial drug residues in milk and meat: cause, concerns, prevalence, regulations, tests, and test performance. J Food Protect 61: 742-756

Navrátilová P, Vyhnálková J, Vorlová L, Jeřábková J 2014: A plate diffusion method for detecting fluoroquinolone residues in raw cow's milk. Czech J Food Sci 32: 260-264

Nouws JFM, Van Egmond H, Loeffen G, Schouten J, Keukens H, Smulders I, Stegeman H 1999: Suitability of the Charm HVS and a microbiological multiplate system for detection of residues in raw milk at EU maximum residue levels. Veterinary Quarterly 21: 21-27

OIE 2018a: OIE annual report on antimicrobial agents intended for use in animals. OIE, Paris, $129 \mathrm{p}$.

OIE 2018b: OIE List of Antimicrobial Agents of Veterinary Importance. Available at: www.oie.int/fileadmin/ Home/eng/Our_scientific_expertise/docs/pdf/AMR/A_OIE_List_antimicrobials_May2018.pdf. Last modified May, 2018. Accessed July 11, 2019

Pikkemaat MG 2009: Microbial screening methods for detection of antibiotic residues in slaughter animals. Anal Bioanal Chem 395: 893-905

Regulation (EC) No. 470/2009 of the European Parliament and of the Council of 6 May 2009 laying down Community procedures for the establishment of residue limits of pharmacologically active substances in foodstuffs of animal origin. OJ L 152: 11-22

Schwarz S, Kehrenberg C, Walsh TR 2001: Use of antimicrobial agents in veterinary medicine and food animal production. Int J Antimicrob Agents 17: 431-437

State Veterinary Administration (SVA) 2008: Methodology for determination of inhibitory substance residues in tissues, eggs, milk and food of animal origin. State Veterinary Institute Jihlava, Czech Republic: 14 p. (in Czech) 
WHO Advisory Group on Integrated Surveillance of Antimicrobial Resistance (AGISAR) 2017: Critically Important Antimicrobials for Human Medicine. Ranking of Medically Important Antimicrobials for Risk Management of Antimicrobial Resistance due to Non-Human Use. $5^{\text {th }}$ revision 2016, Geneva, Switzerland, $41 \mathrm{p}$. 